University of Wollongong

Research Online

Faculty of Informatics - Papers (Archive)

Faculty of Engineering and Information

Sciences

October 2005

\title{
A Protected Digital Item Declaration Language for MPEG-21
}

S. Lauf

University of Wollongong

I. Burnett

University of Wollongong, ianb@uow.edu.au

Follow this and additional works at: https://ro.uow.edu.au/infopapers

Part of the Physical Sciences and Mathematics Commons

\section{Recommended Citation}

Lauf, S. and Burnett, I.: A Protected Digital Item Declaration Language for MPEG-21 2005.

https://ro.uow.edu.au/infopapers/244

Research Online is the open access institutional repository for the University of Wollongong. For further information contact the UOW Library: research-pubs@uow.edu.au 


\title{
A Protected Digital Item Declaration Language for MPEG-21
}

\author{
Abstract \\ Within the MPEG-21 Multimedia Framework, Digital Items are introduced as a structured digital \\ representation for multimedia. To facilitate the representation of Digital Items which include secure or \\ controlled content, the authors have implemented an IPMP Digital Item Declaration Language (IPMP \\ DIDL). This provides for a protected representation of Digital Item structure, allowing the use of existing \\ DRM systems and rights expression languages. This paper examines the design and implementation of \\ the IPMP DIDL and its incorporation into MPEG-21 Part 4: IPMP Components.

\section{Disciplines} \\ Physical Sciences and Mathematics

\section{Publication Details} \\ This paper originally appeared as: Lauf, S \& Burnett, I, A Protected Digital Item Declaration Language for \\ MPEG-21, AXMEDIS 2005. First International Conference on Automated Production of Cross Media \\ Content for Multi-Channel Distribution, 30 October - 2 November 2005, 275-278. Copyright IEEE 2005.
}




\section{A Protected Digital Item Declaration Language for MPEG-21}

\author{
Shane Lauf \\ University of Wollongong \\ shane@jshane.net
}

\author{
Ian Burnett \\ University of Wollongong \\ ianb@uow.edu.au
}

\begin{abstract}
Within the MPEG-21 Multimedia Framework, Digital Items are introduced as a structured digital representation for multimedia. To facilitate the representation of Digital Items which include secure or controlled content, the authors have implemented an IPMP Digital Item Declaration Language (IPMP $D I D L)$. This provides for a protected representation of Digital Item structure, allowing the use of existing DRM systems and rights expression languages. This paper examines the design and implementation of the IPMP DIDL and its incorporation into MPEG-21 Part 4: IPMP Components.
\end{abstract}

\section{MPEG-21 Multimedia Framework}

The MPEG-21 Multimedia Framework has been designed to address the need for interoperable access to content across heterogeneous networks and devices [1]. Digital Items are the currency within the MPEG-21 framework, encapsulating multimedia content and related metadata. The Digital Item Declaration (DID) Model defines a set of entities in Digital Items, and principal examples of these are:

- Resources which link to individually identifiable assets, such as audio or video files

- Components encapsulating and linking metadata with Resources

- Items encapsulating Components and sub-Items

- Containers encapsulating a collection of Items

The Digital Item Declaration Language (DIDL) provides a Representation of the DID Model, allowing the makeup, structure and organization of a Digital Item to be conveyed in a Digital Item Declaration (DID), which is an XML document. DIDL includes elements corresponding to the entities of the DID Model and defined in the normative XML Schema for DID [2]. Figure 1 shows an example Digital Item, which contains a picture and an associated text descriptor, and its representation in DIDL.

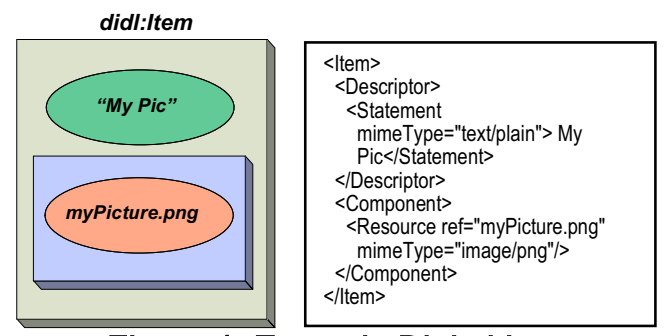

Figure 1: Example Digital Item

Other parts of MPEG-21 include Digital Item Identification (DII), which specifies Identifiers used to identify Digital Items, and parts and collections thereof [3]; the MPEG-21 Rights Expression Language (REL) which expresses the rights, terms and conditions applied to content [4]; and Intellectual Property Management and Protection (IPMP) Components, which provides for the management of rights and intellectual property through the use of protected Digital Items [5].

\section{The need for a protected Digital Item Representation}

One of the goals of Digital Items is that content subject to copyright, privacy controls, and other forms of intellectual property management can be conveyed alongside metadata describing that management. A key issue, however, is that since DIDL is cleartext XML, the possibly sensitive contents of the Digital Item are exposed to view; this allows unauthorized perusal of rights expressions and keys. In response to the pressing need for an alternative, protected Representation of DID Model structure, the authors designed and implemented the IPMP DIDL detailed in this paper.

\section{Design of the MPEG-21 IPMP DIDL}

In designing the IPMP DIDL, the authors recognized the importance of maintaining the Digital Item structure defined in the DID Model, as this structure forms the foundation of MPEG-21 transactions. For 
this reason, work focussed on the design of an alternative Digital Item Representation in XML of that same DID Model structure; this allows Digital Items to be expressed in a protected, but still transactable form.

The solution proposed defines a separate set of protected elements which can be used in a DID document in place of DIDL equivalents and at any point in the Digital Item hierarchy. Thus an equivalent IPMP DIDL element is defined for each DIDL element that corresponds to an entity in the DID Model (e.g. Resource or Item). To protect an existing section of a Digital Item hierarchy expressed in DIDL the XML section is encapsulated in the equivalent IPMP DIDL element, which is then placed back into the Digital Item hierarchy in place of the original DIDL element. Figure 2 shows a DIDL Item being protected in an IPMP DIDL Item.

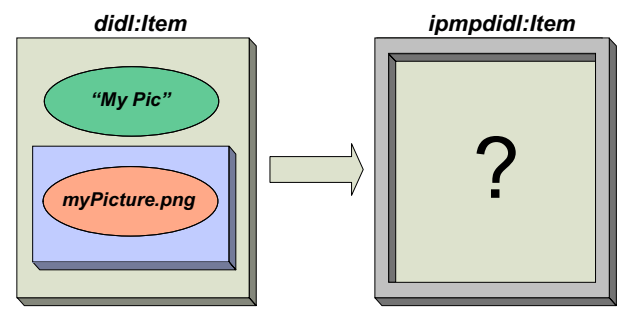

Figure 2: DIDL encapsulation in IPMP DIDL

The key advantage of this method over traditional protection technologies, such as XML encryption, is that an IPMP DIDL representation of a Digital Item remains structurally valid as a transactable Digital Item. This method has been realized in schemas available at http://www.whisper.elec.uow.edu.au/MPEG21/. The design process for these schemas is described below.

\section{Schema design for IPMP DIDL}

For a Digital Item author to protect a specific part of the Digital Item structure by encapsulating it in IPMP DIDL, IPMP DIDL elements must be interchangeable with DIDL equivalents within a DID document. This concept is illustrated in Figure 3, demonstrating how an Item within a Container may be represented as either DIDL (left) or IPMP DIDL (right).

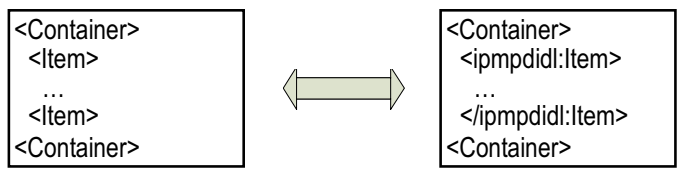

Figure 3: Element interchangeability

For the above scenario to be possible, IPMP DIDL elements must, according to the rules of XML schema, be in the same substitution group as their DIDL equivalents. XML schema substitution holds some parallels with the object-oriented concept of inheritance, and thus the substitutability of element $\mathrm{A}$ for element B may only be declared when $\mathrm{A}$ is an extension of B. The DIDL, however, was not originally designed with such extensibility in mind, and some mechanism for realising element substitutability was necessary. The authors devised and appraised three mechanisms to address this need.

\subsection{Extend and Substitute}

This mechanism realized IPMP DIDL elements as XML extensions (derivations) of the DIDL equivalents, inheriting their characteristics and adding in IPMP-specific structure, as shown in Figure 4.

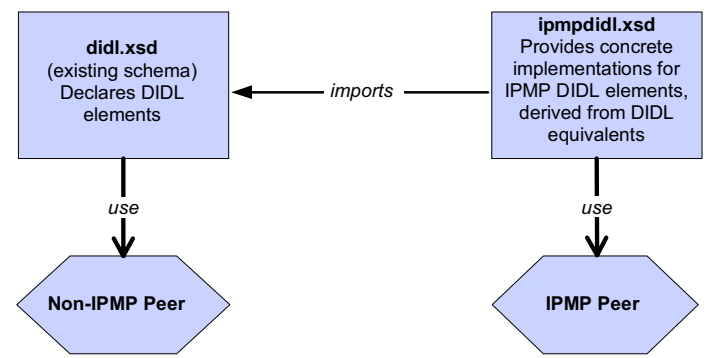

Figure 4: Extend and Substitute mechanism

By virtue of the direct extension from DIDL, this mechanism allowed IPMP DIDL elements to be declared as substitutable for DIDL. However DIDL elements were not originally designed with extension in mind, and may include DIDL-specific mandatory children requirements (for example, didl:Component requires at least one didl:Resource or a didl:Reference child). Thus any IPMP DIDL element which extends these elements also inherits the requirement for these mandatory children, and must contain meaningless 'dummy' children to fulfil schema validity.

\subsection{Separate schema}

An alternative mechanism provided duplicated DIDL schema definitions within an independent IPMP+DIDL schema. In these duplicated definitions, the compulsory child restrictions noted in 4.1 could be removed, avoiding the need for dummy child elements. However this mechanism does not express any interrelationship between DIDL and IPMP DIDL as two expressions of the same DID Model, and requires duplicate definitions in the DIDL and IPMP DIDL schemas to be maintained in parallel. Clearly these are distinct drawbacks for standard schemas where synchronising changes would be necessary. 


\subsection{Abstract derivation}

This third, most innovative solution recognizes that DIDL and IPMP DIDL are both Representations of the DID Model and possess a defined interrelationship, and uses abstract elements to capture the shared semantic. The schema structure is shown in Figure 5.

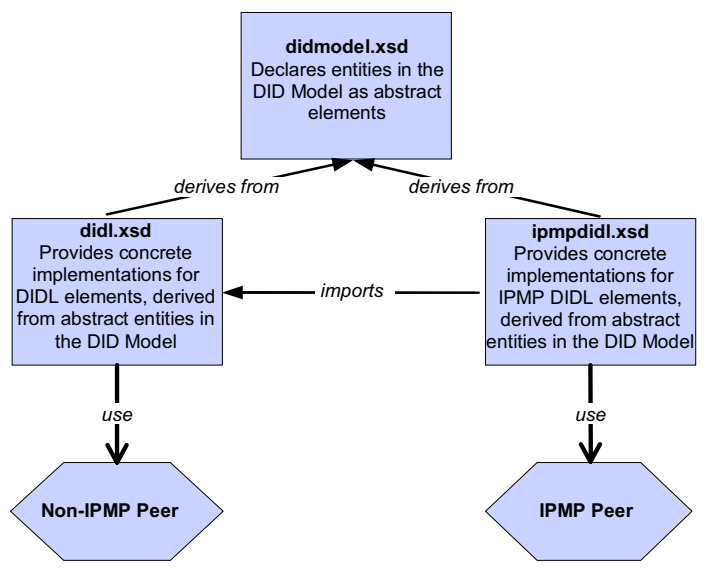

Figure 5: Abstract derivation mechanism

In this mechanism, a parent schema didmodel.xsd represents the DID Model with abstract elements defined to represent each of the DID Model entities (Container, Item, etc.) such as is shown in Figure 6.

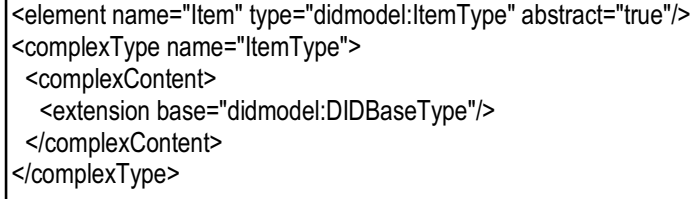

Figure 6: didmodel.xsd abstract element example

Two separate schemas define DIDL and IPMP DIDL elements, derived from the parent DID Model elements and with structure appropriate to each Representation, and place them in substitution groups with the parent. Where DIDL elements have child elements, these are declared in terms of the abstract elements. This is shown in Figure 7 and Figure 8.

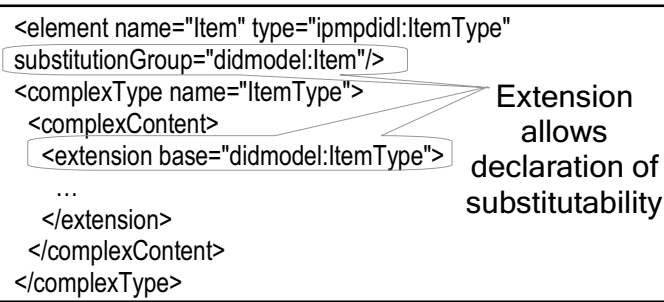

Figure 7: ipmpdidl.xsd element example

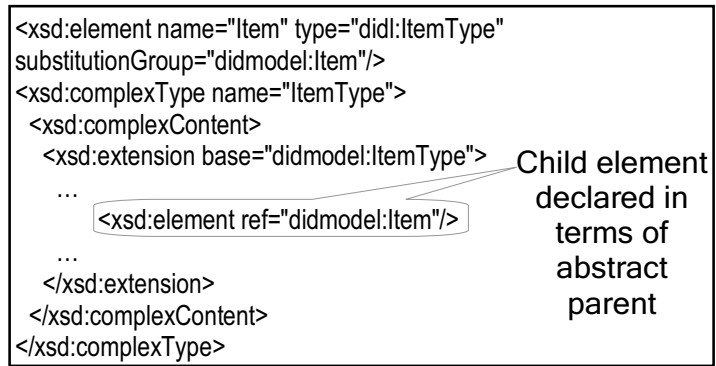

Figure 8: didl.xsd element example

\subsection{Evaluation}

The strengths and weaknesses of each mechanism approach were examined, as shown in Table 1.

Table 1: Evaluation of schema mechanisms

\begin{tabular}{|l|c|c|c|}
\hline \multicolumn{1}{|c|}{ Mechanism } & $\begin{array}{c}\text { Extend } \\
\text { and } \\
\text { Substitute }\end{array}$ & $\begin{array}{c}\text { Separate } \\
\text { schema }\end{array}$ & $\begin{array}{c}\text { Abstract } \\
\text { Derivation }\end{array}$ \\
\hline $\begin{array}{l}\text { IPMP DIDL } \\
\text { substitutable for } \\
\text { DIDL }\end{array}$ & $\boldsymbol{X}$ & $\boldsymbol{V}$ & \\
\hline $\begin{array}{l}\text { No mandatory } \\
\text { children on IPMP } \\
\text { DIDL }\end{array}$ & $\boldsymbol{X}$ & $\mathbf{V}$ \\
\hline $\begin{array}{l}\text { No duplicate } \\
\text { definitions requiring } \\
\text { synchronization }\end{array}$ & & $\mathbf{X}$ & \\
\hline
\end{tabular}

The authors selected the abstract derivation mechanism as the most flexible means to facilitate DIDL and IPMP DIDL element interchangeability within a protected DID document, allowing DIDL and IPMP DIDL to be different, schematically valid Representations of the DID Model.

\section{Structure of IPMP DIDL elements}

Once the substitution of IPMP DIDL elements for DIDL equivalents was resolved, the second challenge was the design of the internal structure for these IPMP DIDL elements. Since these elements encapsulate protected DIDL structure alongside relevant information about the protection, the structure was implemented as shown in Figure 9.

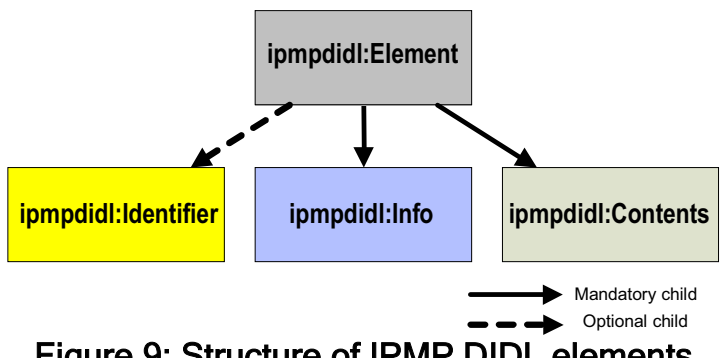

Figure 9: Structure of IPMP DIDL elements 
Each of the sub-elements constituting this structure is considered in more detail below.

- Identifier: Contains an identifier from an appropriate identification scheme (such as DII) which may be used, for example, when a REL Grant needs to refer to the protected content about which it expresses rights.

- Info: Contains expression of mechanisms and licenses involved in the protection of and access to content. This element may link to an existing DRM.

- Contents: Contains the governed DIDL structure or asset itself, possibly with encryption, watermarking or other protection tools applied.

Using this three-element structure facilitates the encapsulation and protection of DIDL structure in IPMP DIDL elements with structure as shown in Figure 10.

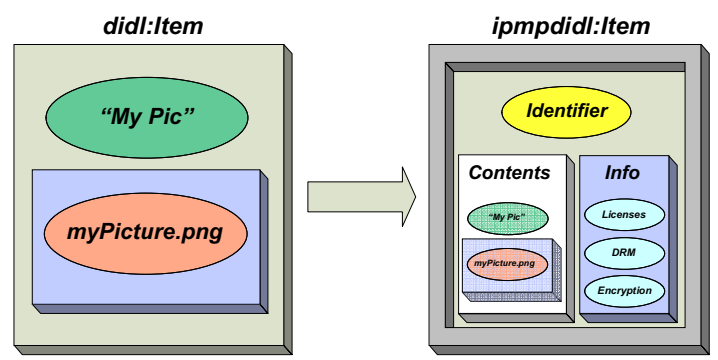

Figure 10: DIDL encapsulation in IPMP DIDL

For particularly sensitive Digital Item contents, the ipmpdidl:Contents may be encrypted or otherwise protected, with a tool described in ipmpdidl:Info. In this case the structure and contents of ipmpdidl:Contents will not be visible, as shown in Figure 11.

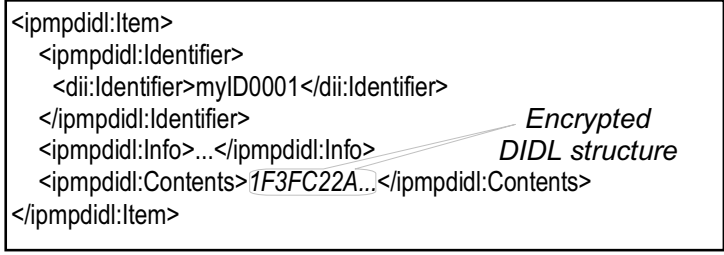

Figure 11: Digital Item structure encryption

\section{Implementation}

Following the design of schemas according to the mechanisms decribed above, the authors sought to confirm the feasibility of the IPMP DIDL thus defined through the implementation of a prototype MPEG-21 Peer. This prototype was developed as a Java-based mobile phone application, subject to the strict memory and processing limitations of the MIDP 2.0 Profile of Java 2 Mobile Edition. This mobile Peer was successfully implemented, and parses protected Digital Items according to the IPMP DIDL schema. It also incorporates other IPMP and REL technology to demonstrate the unlocking of protected Digital Item structure according to associated information about the protection mechanisms, and contingent on the validity of associated digital rights expressions.

This implementation confirmed the usefulness of the IPMP DIDL in representing and transacting Digital Items in protected form even on limited platforms. As a result the IPMP DIDL has been adopted into the MPEG-21 Part 4: IPMP Components standard.

\section{Conclusion}

This paper has described the realization of IPMP DIDL as a protected Representation of the MPEG-21 DID Model. The work has contributed a complete schema solution that facilitates the expression and processing of this protected Representation, and demonstrated its usefulness in an IPMP-enabled MPEG-21 Peer. As a result, Digital Items may include protected structure and information about that protection, allowing Digital Item authors to leverage existing DRM and REL technology. The IPMP DIDL is a simple yet innovative solution that maintains the concept, structure and transactability of the Digital Item while significantly increasing its usefulness in a DRM-hungry multimedia world.

\section{References}

[1] Information Technology - Multimedia Framework Part 1: Visions, Technology and Strategy TR, ISO/IEC JTC1/SC29/WG11/N6388, March 2004, Munich

[2] R. Van de Walle, I. Burnett, G. Drury (editors), ISO/IEC 21000-2 DID 2nd Edition FDIS, ISO/IEC JTC1/SC29/WG11/N6927, January 2005, Hong Kong

[3] Information Technology - Multimedia Framework Part 3: Digital Item Identification, ISO/IEC JTC1/SC29/WG11/N4939, July 2002, Klagenfurt

[4] Information Technology - Multimedia Framework - Part 5: Rights Expression Language, ISO/IEC JTC1/SC29/WG11/N5839, July 2003, Trondheim

[5] S. Watt, S. Lauf, Z. Huang, E. Rodriguez (editors), ISO/IEC 21000-4 IPMP Components FCD, ISO/IEC JTC1/SC29/WG11/N7196, April 2005, Busan

[6] W3C, XML Schema, May 2001. http://www.w3.org/TR/2001/

[7] Sun Microsystems, Mobile Information Device Profile $\begin{array}{lllll}\text { (MIDP) } & \text { JSR } & 37, & \text { JSR } & 118 .\end{array}$ http://java.sun.com/products/midp/index.jsp 